\title{
Observation of fluid flow changes at low velocities interval by adaptive photodetection
}

\author{
Observación de cambios de flujo en fluidos en intervalo de bajas \\ velocidades por fotodetección adaptiva
}

\author{
M. Martínez Ayala(A), D. Mayorga Cruz( $\left.{ }^{*}, A\right), F$. Z. Sierra Espinosa \\ Centro de Investigación en Ingeniería y Ciencias Aplicadas, UAEM. Avenida Universidad 1001, Colonia Chamilpa, C.P. \\ 60209, Cuernavaca Morelos, México

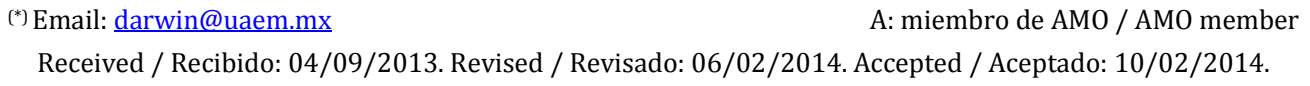

\begin{abstract}
:
In this work a brief experimental investigation of velocity changes on a fluid flow by means of a noncontactive optical method based on the Non-steady-state photo-electromotive force (P-EMF) effect is presented. Evidence of linear dependence of P-EMF signal on fluid flow for low velocities as compared with a Laser Doppler Anemometry test is shown and discussed.
\end{abstract}

Key words: Non-Contactive Measurements, Laser Velocimetry, Adaptive Photodetectors.

\section{RESUMEN:}

En este trabajo se presenta una breve investigación experimental sobre los cambios de velocidad en un flujo de fluido por medio de un método óptico no contactivo basado en el efecto de fuerza fotoelectromotriz no estacionario. Se muestra evidencia de una dependencia lineal de la señal P-EMF con respecto al flujo de fluido para bajas velocidades.

Palabras clave: Mediciones No Contactivas, Velocimetría Láser, Fotodetectores Adaptivos.

\section{REFERENCES AND LINKS / REFERENCIAS Y ENLACES}

[1]. Fluid Meters. Their Theory and Application, Report of ASME, Research Committee on Fluid Meters, New York, Publishers by ASME (1971).

[2]. Y. Yeh, H. Z. Cummins, "Localized fluid flow measurements with a He-Ne Laser spectrometer", Appl. Phys. Lett. 4, 176-178 (1964). DOI

[3]. H. E. Albretch, Laser Doppler and Phase Doppler Measurement Technique. Springer, New York (2003). DOI

[4]. S. Stepanov. "Photo-electromotive-force effect in semiconductors", in Handbook of Advanced Electronic and Photonic Materials and Devices, Academic Press (2001). DOI

[5]. M. P. Petrov, I. A. Sokolov, S. I. Stepanov, G. S. Trofimov. "Non-steady-state photo-electromotive force induced by dynamic gratings in partially compensated photoconductors", J. Appl. Phys. 68, 2216-2225 (1990). DOI

[6]. C. C. Wang, F. Davidson, S. Trivedi. "Simple laser velocimetry that uses photoconductive semiconductors to measure optical frequency differences", Appl. Opt. 34, 6496-6499 (1995). DOI

[7]. C. C. Wang, S. Trivedi, F. Jing, K. Jia, H. He, G. S. Elliott, J. Khurgin. "High-precision measurement of optical frequency differences between Q-switched laser pulses using photo-electromotive-force sensors", J. Opt. Soc. Am. B 19, 177-182 (2002). DOI

[8]. S. Muñoz Solís, M. L. Arroyo Carrasco, M. M. Méndez Otero, E. Martí Panameño, P. Rodríguez Montero. "Measurement of fluid velocities by means of photo-electromotive force effect", Conference Paper JWB20, Optical Amplifiers and Their Applications (OAA), Optical Society of America (2006). 
[9]. M. Boguszko, G. S. Elliott, C.C. Wang, S. Trivedi, S. Tse, J.F. Meyers, J. Lee. "Fluid flow Doppler velocimetry using adaptive photo-induced electromotive force detectors", 43th AIAA Aerospace Sciences Meeting and Exhibit, Conference Paper p. 1-14, Paper AIAA 2005-0625 (2005).

\section{Introduction}

Flow velocity measurement is an important topic for several basic research and technological areas, as an inseparable part of flow chart processes [1]. Sometimes under certain physical conditions it is advisable not to disturb flow evaluations and then noncontactive optical inspection methods are recommendable options as these are based on specular or backscattered reflection light coming from the object under inspection, which can be processed and analyzed in order to "unwrap" the information related with mechanical surface (or volume) information. Among several forward scatter and backscatter geometries methods, Laser Doppler anemometry (LDA) have shown the most outstanding performance and is commonly used in almost every fluid mechanics laboratory in the world, because of its capacity to measure the velocity of fluids by observing the Doppler shift in the frequency of light scattered from small particles moving with the fluid [2,3]. However, specialized electronic and signal processing instrumentation used to perform LDA measuring complicates its applicability for in situ measurements; then the needing to apply simpler or complementary techniques to LDA to achieve fluids velocity measurements is clearly evident.

Recently, adaptive photodetection, a relatively new optoelectronic method have been used as an alternative technique for several applications as remote measurement of surface vibrations, analysis of modulated optical signal power spectrum, phase-locking of separated lasers, or characterization of photonic parameters related to photoconductive materials [4]. An adaptive photodetector is a device based on the photo-electromotive force effect (P-EMF), which consists on the appearance of an alternating current in a photoconductive material, a semiconductor crystal for example, illuminated by a regular (interference) or irregular (speckle) oscillating optical pattern of varying intensity [5]. The capacity of adaptive photodetectors to perform remote detection of velocity by means of Doppler frequency shift between the probe and signal beams is a quite interesting application for aerodynamics and fluid mechanics, and possibilities for velocimetry of moving solid objects on velocity range up to $0.4 \mathrm{~mm} / \mathrm{s}$ have been previously shown [6,7]. Lately, flow velocities measuring obtained from the optical interference of backscattered light from a fluid with a phase-modulated beam, or a subsonic free jet were performed at higher velocity intervals ranging from 100 to $800 \mathrm{~cm} / \mathrm{s}$ [8,9]. Here, an analysis for a water-fluid at a lower velocity range using a P-EMF configuration and a comparison with well established LDA technique in order to explore adaptive photodetection as an eventual alternative for flow velocimetry, is presented.

\section{Theory}

When two radiant beams are intersected on the surface of a photoconductive material sample at an angle $\alpha$, an interference pattern consisting on bright and dark fringes appears. On bright zones an excess charge carriers is produced and tend to migrate to the dark zones, where the spacecharge redistribution is generated and produces in turn an electric space-charge field Esc; if no external electric field is applied, $E_{s c}$ is $\pi / 2$ phase shifted with respect to the photoconductivity pattern $\sigma_{0}$, and no current will be flowing through the sample. However, when one of the beams is frequency-modulated due to an external perturbation (i.e. Doppler frequency shift from a fluid flow), the fringe pattern will move with an angular frequency difference $\Omega$ given by the perturbation. It allows the photoconductivity $\sigma_{0}$ be periodically in phase with respect to Esc and an electric photocurrent is generated through the volume of the sample due to the Ohm law.

When particles moving within a fluid flowing in an specific direction are illuminated by an external optical source, resulting Doppler 
angular frequency shift produced by reflected light is given by:

$$
\Omega=\frac{2 \pi v}{\lambda}
$$

where $\lambda$ is the optical source wavelength and $v$ the velocity of the moving particle in the flow direction. If an optical pattern produced by interference of reflected beam with a reference beam coming from the same source illuminates the sample [9]:

$$
I(x)=I_{0}[1+m \cos (K x-\Omega t)],
$$

with average radiant intensity $I_{0}=I_{S}+I_{R}$, fringe pattern contrast

$$
m=\frac{2 \sqrt{I_{S}+I_{R}}}{I_{S}+I_{R}}
$$

and spatial frequency

$$
K=\frac{4 \pi \sin \alpha}{\lambda}
$$

the expression of generated P-EMF current density for a monopolar semiconductor $[4,5,9]$ with small contrast $(m<<1)$, no external DC field applied, no saturation of impurity centres and a running interference pattern speed $v=\Omega / K$ is:

$$
j_{p E M F}=\frac{m^{2} \sigma_{0} E_{D} \Omega \tau_{d}}{2\left\{1+\left[\Omega \tau_{d}\left(1+K^{2} L_{D}^{2}\right)\right]^{2}\right\}} .
$$

Here $\sigma_{0}$ is the average photoconductivity, $E_{D}=K k_{B} T / e$ the diffusion electric field, $\tau_{d}$ the mobile carrier dielectric relaxation time and $L_{D}$ the diffusion length. Several adaptive photodetectors applications, as flow velocimetry for example, may seem feasible mainly due to their sensitivity on optical intensity and contrast changes (Eq.(5)).

\section{Experimental setup}

The experimental setup shown at Fig. 1 is based on a modified Twyman-Green interferometer to generate P-EMF configuration. A low power (75 $\mathrm{mW})$ He-Ne laser beam $(\lambda=632.8 \mathrm{~nm})$ is divided with one arm directed to a transparent walls tube system, where water fluid carrying polystyrene microparticles is cycling at the 1-40 $\mathrm{cm} / \mathrm{s}$ range; scattered light from particles is focused onto the surface of a GaAs based

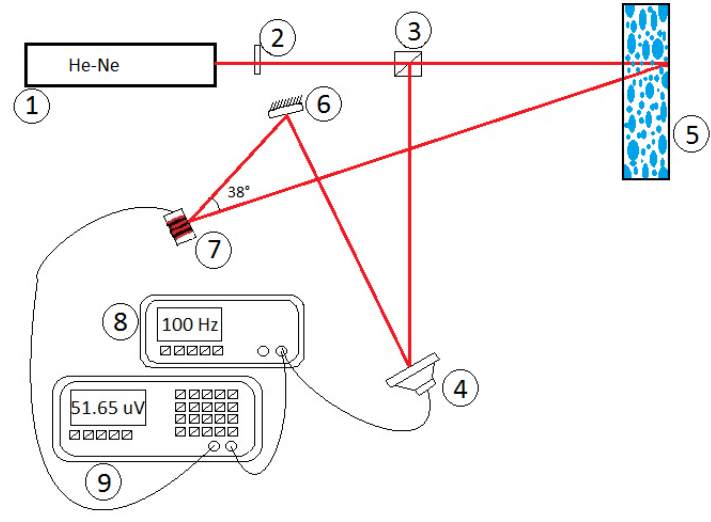

Fig. 1. Experimental setup. 1: He-Ne laser, 2: logarithmic filter, 3: beamsplitter, 4: vibrating mirror, 5: fluid flow system, 6: mirror, 7: GaAs photodetector, 8: signal generator, 9: lock-in amplifier.

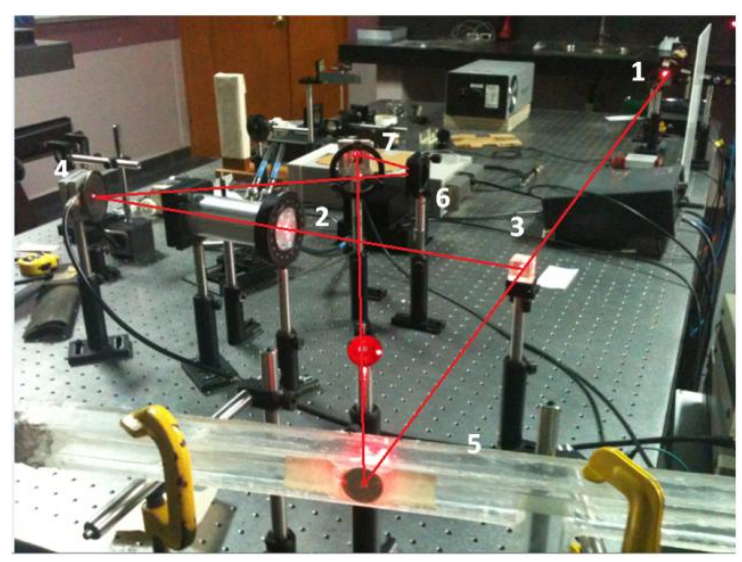

Fig. 2. Photograph of setup showing the elements described on Fig. 1.

adaptive photodetector at a $P_{S}=12.4 \mu \mathrm{W}$ in the front of the crystal, where it interferes with the phase-modulated second arm reflected from a mirror on a loudspeaker fed with a signal generator at modulation frequency values $\Omega / 2 \pi$ and a $P_{R}=5.4 \mu \mathrm{W}$ measured also in the front of the crystal, at a common $\theta=38^{\circ}$ between the beams. The generated P-EMF output voltage signal $U^{\Omega}$, measured through the silver paint contacts on the crystal is monitored by a lock-in amplifier also connected to the signal generator with an electric reference, and experimental data are manually registered and post-analyzed. A photograph of experimental setup shows the simplicity of this optical method (Fig. 2). 


\section{Results}

On Fig. 3(a), the P-EMF signal dependences as a function of fluid flow velocity measured for three different $\Omega / 2 \pi$ modulation frequencies are illustrated.

As it can be seen, P-EMF signal dependences showed no significant changes with respect to flow velocity changes at tested frequencies, as should be expected; a quasi-constant flow rate system like the one here considered usually present typical linear responses, as such given by well-established optical methods like laser Doppler anemometry (LDA) [3]. On the other hand, Fig. 3(b) shows a linear-like dependence of P-EMF signal on flow velocities at a lower modulation frequency $(100 \mathrm{~Hz})$; the simple linear regression data fitting performed with an application software gives a coefficient of determination $R^{2}=0.8842$ stating the observed behavior.

Such a dependence may be related with running interference pattern speed $v$, because it is well known there is a characteristic time directly related to mobile carrier dielectric relaxation time $\left(\tau_{d}\right)$ for space-charge distribution (responsible of P-EMF signal $U^{\Omega}$ ). At high speeds $\left(\Omega \ll \tau_{d}^{-1}\right)$ fringes move so fast giving free charges not enough time to diffuse and form the space-charge, and as a consequence it cannot follow the fringe pattern and output P-EMF signal is not linear.

Following this last consideration modulation frequencies range $\Omega<100 \mathrm{~Hz}$ were tested for PEMF signal dependences and, additionally laser Doppler anemometry tests were also performed in the fluid system at the same flow velocities, in order to observe P-EMF $U^{\Omega}$ response at lower frequencies as compared to the LDA signal one (Fig. 4).

It can be seen how around $\Omega / 2 \pi=50 \mathrm{~Hz}$, a linear-type P-EMF signal response is obtained compared to the typical LDA responses, as expected (Fig. 4(b)). Although slopes of the two straight lines are clearly not the same for both dependences, this apparent discordance may be explained due to the fact that LDA data are related with a central position of flow trajectory along the squared tube, which is not the case for the P-EMF signal measured at certain point close to the wall; the possibility to perform adaptive photodetection by using short coherent length sources, as LED or super-luminescent diodes for a specific localized measure of fluid velocity inside the flow may be a suitable alternative [8].
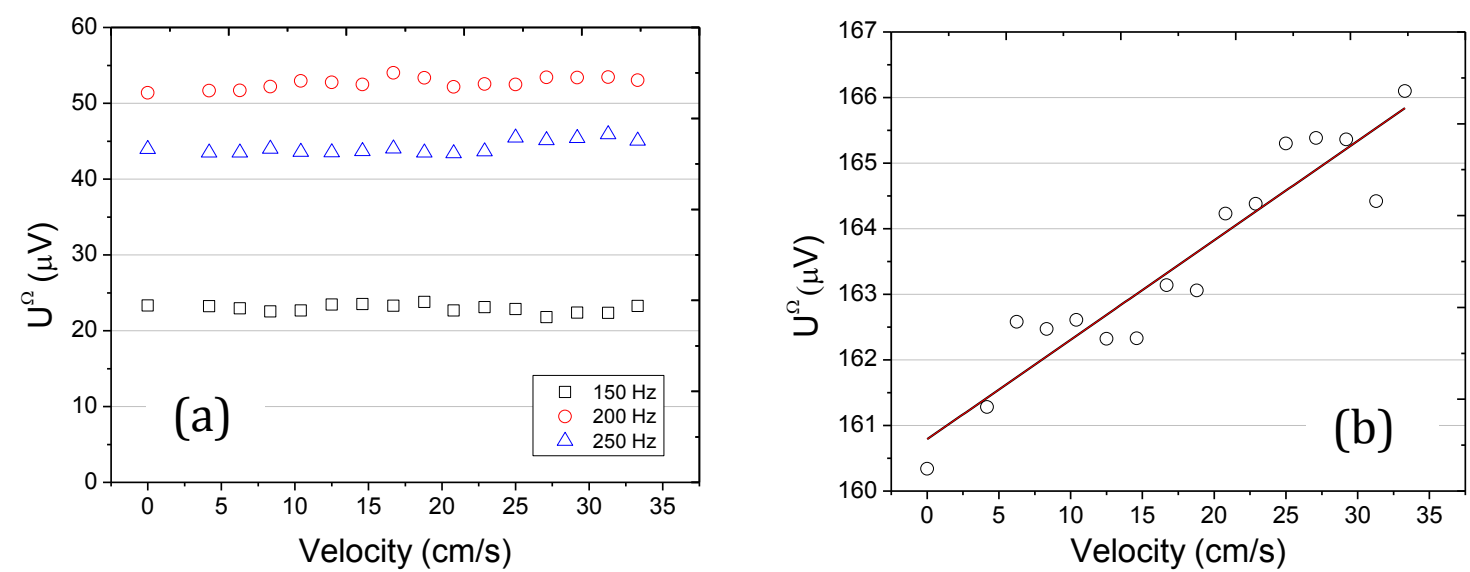

Fig. 3. (a) P-EMF signal $U^{\Omega}$ as a function of fluid velocity measured in the fluid flow for three different specific modulation frequencies; b) P-EMF signal $U^{\Omega}$ as a function of fluid velocity measured in the fluid flow at $\Omega / 2 \pi=100 \mathrm{~Hz} ; R^{2}=0.8842$ is the coefficient of determination obtained by linear data fitting. 
(a)

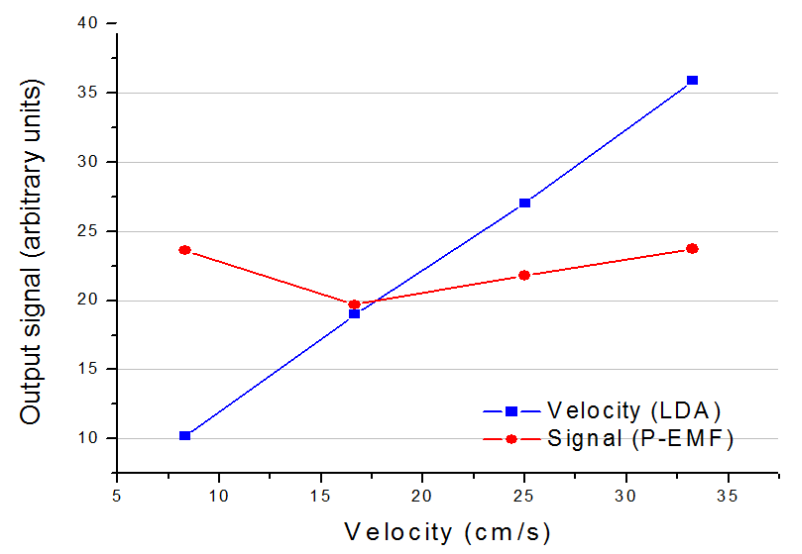

(c)

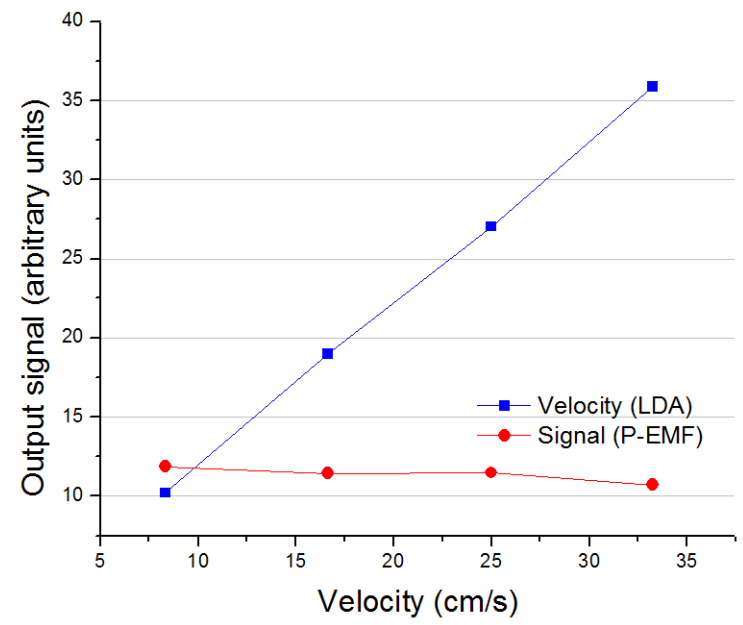

(b)

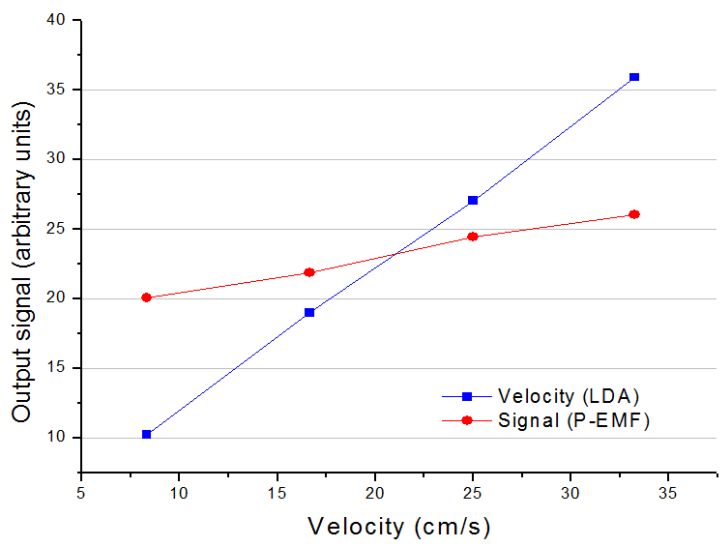

(d)

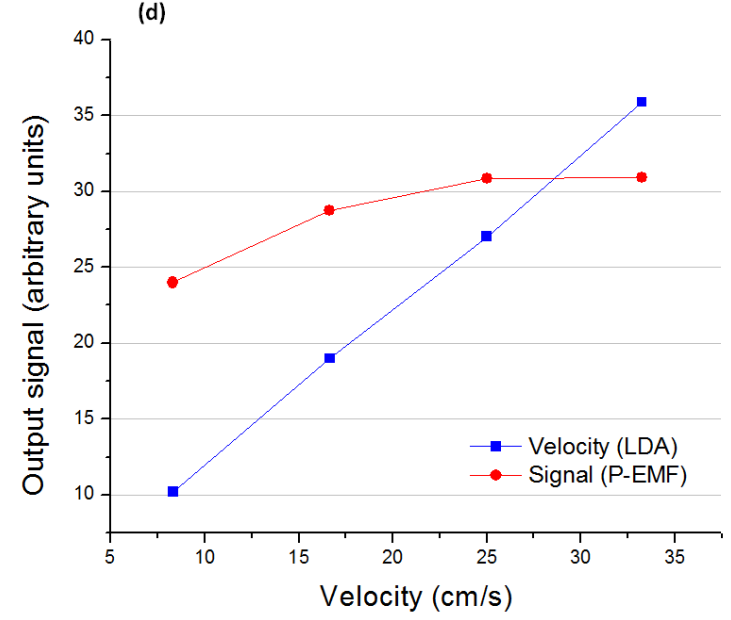

Fig. 4. P-EMF and LDA output signals as a function of fluid velocity measured in the fluid flow for different P-EMF modulation frequency values. (a) $\Omega / 2 \pi=40 \mathrm{~Hz}$; (b) $\Omega / 2 \pi=50 \mathrm{~Hz}$; (c) $\Omega / 2 \pi=70 \mathrm{~Hz}$; (d) $\Omega / 2 \pi=100 \mathrm{~Hz}$.

\section{Conclusions}

Our results may suggest the possibility to explore lower modulation P-EMF frequencies, within the linear dependence growth regime of $U^{\Omega}$, in order to find more stable conditions to obtain accurate measuring for reliable signalfluid velocity experiments; preliminary comparison of non-steady-state P-EMF with laser Doppler anemometry techniques confirms a linear dependence, typical of quasi-constant flow rate systems. Further comparative analysis of both methods should be complemented with numerical calculation of the scaling factor in order to establish adaptive photodetection as a practical tool for fluid velocimetry and other similar applications.

\section{Acknowledgments}

Mauricio Martínez Ayala acknowledges the National Council on Science and Technology (CONACYT) for a grant scholarship. 